\title{
The Antimalarial Effect of Curcumin Is Mediated by the Inhibition of Glycogen

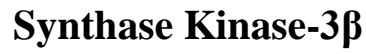

\begin{abstract}
Curcumin, a bioactive compound in Curcuma longa, exhibits various pharmacological activities, including antimalarial effects. In silico docking simulation studies suggest that curcumin possesses glycogen synthase kinase-3 $\beta$ (GSK3 $\beta$ )-inhibitory properties. The involvement of GSK3 in the antimalarial effects in vivo is yet to be demonstrated. In this study, we aimed to evaluate whether the antimalarial effects of curcumin involve phosphorylation of host GSK3 $\beta$. Intraperitoneal administration of curcumin into Plasmodium berghei NK65-infected mice resulted in dose-dependent chemosuppression of parasitemia development. At the highest dose tested $(30 \mathrm{mg} / \mathrm{kg}$ body weight), both therapeutic and prophylactic administrations of curcumin resulted in suppression exceeding $50 \%$ and improved median survival time of infected mice compared to control. Western analysis revealed a 5.5-fold (therapeutic group) and 1.8-fold (prophylactic group) increase in phosphorylation of Ser 9 GSK3 $\beta$ and 1.6-fold (therapeutic group) and 1.7-fold (prophylactic group) increase in Ser 473 Akt in liver of curcumin-treated infected animals. Following P. berghei infection, levels of pro- and anti-inflammatory cytokines, tumor necrosis factor (TNF)- $\alpha$, interferon (IFN)- $\gamma$, interleukin (IL)-10, and IL-4 were elevated by 7.5-, 35.0-, 33.0-, and 2.2-fold, respectively. Curcumin treatment (therapeutic) caused a significant decrease (by 6.0- and 2.0-fold, respectively) in serum TNF- $\alpha$ and IFN- $\gamma$ level, while IL-10 and IL-4 were elevated (by 1.4- and 1.8-fold). Findings from the present study demonstrate for the first time that the antimalarial action of curcumin involved inhibition of GSK3 $\beta$.
\end{abstract}

Keyword: Curcumin; In vivo; Inflammation; Malaria; Nuclear factor-kappa B (NF-кB) 